\title{
The Distribution of Maximal Prime Gaps in Cramér's Probabilistic Model of Primes
}

\author{
Alexei Kourbatov ${ }^{1}$ \\ ${ }^{1}$ JavaScripter.net, Redmond, Washington, USA \\ Correspondence: Alexei Kourbatov, 15127 NE 24th Street \#578, Redmond, WA 98052, USA. E-mail: \\ akourbatov@gmail.com
}

Received: February 7, 2014 Accepted: March 11, 2014 Online Published: March 20, 2014

doi:10.5539/ijsp.v3n2p18 URL: http://dx.doi.org/10.5539/ijsp.v3n2p18

\begin{abstract}
In the framework of Cramér's probabilistic model of primes, we explore the exact and asymptotic distributions of maximal prime gaps. We show that the Gumbel extreme value distribution $\exp (-\exp (-x))$ is the limit law for maximal gaps between Cramér's random "primes". The result can be derived from a general theorem about intervals between discrete random events occurring with slowly varying probability monotonically decreasing to zero. A straightforward generalization extends the Gumbel limit law to maximal gaps between prime constellations in Cramér's model.
\end{abstract}

Keywords: distribution of primes, prime gap, prime $k$-tuple, prime constellation, extreme value theory, Cramér conjecture, Gumbel distribution, asymptotic distribution, Hardy-Littlewood $k$-tuple conjecture

2010 Mathematics Subject Classification: Primary 11N05; Secondary 60G70, 62E20

\section{Introduction}

In this paper we apply extreme value theory to Cramér's probabilistic model of primes. But first let us say a few words about two mathematicians who pioneered these important topics. The Swedish mathematician Harald Cramér (1893-1985) made long-lasting contributions in statistics and number theory. His model of primes continues to serve as a heuristic tool leading to new insights in the distribution of primes. Cramér wrote:

In investigations concerning the asymptotic properties of arithmetic functions, it is often possible to make an interesting heuristic use of probability arguments. If, e. g., we are interested in the distribution of a given sequence $S$ of integers, we then consider $S$ as a member of an infinite class $C$ of sequences, which may be concretely interpreted as the possible realizations of some game of chance. It is then in many cases possible to prove that, with a probability $=1$, a certain relation $R$ holds in $C$, i. e., that in a definite mathematical sense "almost all" sequences of $C$ satisfy $R$. Of course we cannot in general conclude that $R$ holds for the particular sequence $S$, but results suggested in this way may sometimes afterwards be rigorously proved by other methods. (Cramér, 1936, p. 25)

It is difficult to ascertain whether Harald Cramér had ever met in person his contemporary, the German-American mathematician Emil Julius Gumbel (1891-1966), one of the founders of extreme value theory. This branch of statistics is used today for describing phenomena in vastly diverse areas, ranging from actuarial science to hydrology to number theory. In Statistics of Extremes (1958) Gumbel observed:

... many engineers and practical statisticians ... are inclined to believe that, after all, nearly everything should be normal, and whatever turns out not to be so can be made normal by a logarithmic transformation. This is neither practical nor true. (Gumbel, 1958, p. 345)

In Les valeurs extrêmes des distributions statistiques (1935) Gumbel showed that extreme values taken from a sequence of i.i.d. random variables with an exponential distribution obey the double exponential limit law (now known as the Gumbel distribution). He reconfirmed the earlier result of Fisher and Tippett (1928) that the same limit law also holds for extreme values of i.i. d. random variables with a normal distribution - and generalized it to a much wider class of initial distributions, the so called exponential type. To wit: 
Pour les valeurs extrêmes ... on arrive à la distribution doublement exponentielle pourvu que la distribution initiale appartienne au type exponentiel ... Cette theorie est susceptible de nombreuses applications, puisque en particulier, la distribution de Gauss et la distribution exponentielle appartiennent au type exponentiel (Gumbel, 1935, p. 154-155).

We will draw upon the work of both Cramér and Gumbel: In Section 4.2 we show that the limiting distribution of maximal prime gaps in Cramér's probabilistic model is the Gumbel extreme value distribution. In hindsight, the result is not very surprising: we know from computation and distribution fitting that the actual maximal prime gaps are indeed nicely approximated by the Gumbel distribution (Kourbatov, 2013) (What is somewhat surprising is that Cramér or Gumbel have not themselves published a similar result long ago, perhaps in the 1930s or 1940s. Did they possibly deem it obvious? We might never know).

The existence of the Gumbel limit law for maxima of the (non-identically distributed) gaps between Cramér's random primes is interesting in view of Mejzler's theorem (see, e.g., de Haan \& Ferreira, 2006, p. 201). For extremes of non-identically distributed independent random variables, Mejzler's theorem states that the limiting distribution (if it exists at all) can be any distribution with a log-concave cdf. Thus, prime gaps in Cramér's model give us an example of non-identically distributed random variables whose extremes nevertheless possess a limit law that is allowed in i.i.d. situations as well.

\section{Definitions • Notations • Abbreviations}

\begin{tabular}{|c|c|}
\hline a.s. & almost sure, almost surely \\
\hline i.i.d. & independent and identically distributed \\
\hline cdf & cumulative distribution function \\
\hline pdf & probability density function \\
\hline Ex & the expected value (mathematical expectation) of the random variable $x$ \\
\hline $\operatorname{Exp}(x ; a)$ & the exponential distribution cdf: $\operatorname{Exp}(x ; a)=1-e^{-x / a}$ \\
\hline $\operatorname{Gumbel}(x ; a, \mu)$ & the Gumbel distribution cdf: $\operatorname{Gumbel}(x ; a, \mu)=e^{-e^{-\frac{x-\mu}{a}}}=2^{-e^{-\frac{x-M}{a}}}$ \\
\hline$a$ & the scale parameter of exponential/Gumbel distributions, as applicable \\
\hline$\mu$ & the location parameter (mode) of the Gumbel distribution \\
\hline$M$ & the median of $\operatorname{Gumbel}(x ; a, \mu): M=\mu-a \log \log 2 \approx \mu+0.3665 a$ \\
\hline $\mathrm{RP}$ & a random "prime" in the context of Cramér's model (a white ball) \\
\hline $\mathrm{RC}$ & a random "composite" in the context of Cramér's model (a black ball) \\
\hline$p_{k}$ & the $k$-th prime; $\left\{p_{k}\right\}=\{2,3,5,7,11, \ldots\}$ \\
\hline$P_{k}$ & the $k$-th random "prime" (RP) in Cramér's model \\
\hline$U_{n}$ & the $n$-th urn producing RPs with probability $\frac{1}{\log n}$ in Cramér's model \\
\hline$R_{n}$ & a random variable: the longest uninterrupted run of $\mathrm{RCs} \leq n$ \\
\hline$G_{n}$ & a random variable: the maximal gap between $\operatorname{RPs} \leq n ; \quad G_{n}:=R_{n}+1$ \\
\hline$\pi(x)$ & the prime counting function, the total number of primes $p_{k} \leq x$ \\
\hline$\Pi(x)$ & the RP counting function, the total number of RPs $P_{k} \leq x$ \\
\hline $\log x$ & the natural logarithm of $x$ \\
\hline $\operatorname{li} x$ & the logarithmic integral of $x$ : $\operatorname{li} x=\int_{0}^{x} \frac{d t}{\log t}=\int_{2}^{x} \frac{d t}{\log t}+1.04516 \ldots$ \\
\hline
\end{tabular}

\subsection{Definitions of Gaps and Runs}

Prime gaps are distances between two consecutive primes, $p_{k}-p_{k-1}$ (OEIS A001223, Sloane, 2014). In the context of Cramér's model (see next section) "prime" gaps refer to distances between consecutive RPs, $P_{k}-P_{k-1}$.

Maximal gaps between primes are usually defined as gaps that are strictly greater than all preceding gaps (OEIS A005250, Sloane, 2014). However, for Cramér's model, we will use the term maximal gap in the statistical sense defined below. Note that Cramér's model does not guarantee that there are any "primes" $P_{k}>2$ at all. To make sure that maximal gaps are defined in all cases, we first define the longest run of random "composites" (RCs) $\leq n$ :

$$
R_{n}=\text { the longest run of consecutive RCs } \leq n \text { (allowing runs of length } 0 \text { ). }
$$


We now define the maximal gap between RPs $\leq n$ simply as

$$
G_{n}=R_{n}+1 .
$$

Compare our definition of $G_{n}$ to the definition of maximal prime gaps for true primes:

$$
\text { maximal prime gap up to } n=\max _{p_{k} \leq n}\left(p_{k}-p_{k-1}\right) \text {. }
$$

Clearly, the same gap/run relation holds (with very rare exceptions) for true primes as well:

$$
\text { maximal prime gap }=1+\text { the longest run of composites below } n \text {. }
$$

Examples: the largest prime gap below 100 is the gap of 8-between the primes 89 and 97; this corresponds to a run of 7 consecutive composites: 90 to 96 . Exceptional cases ( $g a p \neq 1+$ run) occur towards the end of record prime gaps. Consider, e. g., the prime gap $113 \ldots 127$ and take $n=125$. Then the largest prime gap below $n$ is still the gap of 8-between 89 and 97-while the longest run of 12 composites $\leq n$ occurs from 114 to 125 .

Of course, in the probabilistic model, both $R_{n}$ and $G_{n}$ are random variables and, by definition, $G_{n}=R_{n}+1$ without exceptions. In Section 4 we will investigate the distribution of maximal gaps $G_{n}$.

\subsection{Remark: Maxima vs. Records}

Number theorists may use the terms maximal gaps and record gaps as synonyms (Caldwell, 2010; Nicely, 2013) while statisticians make a distinction between maximal and record values (Arnold et al., 1998; Nevzorov, 2001). Resnick (1973, Theorem 8) shows that the distinction is quite profound: in the i.i.d. case, the limit laws for records and maxima cannot be the same. Nevertheless, for a wide class of sequences of non-identically distributed random variables (the $F^{\alpha}$ model) the same extreme value distribution (e.g. Gumbel distribution) can be the limit law for both records and maxima (Arnold et al., 1998, p. 193). Clearly, Cramér's "prime" gaps near, say, urn $U_{10}$ are distributed not identically to those near urn $U_{100}$. Theorem 1 (Sect. 4.2) establishes that the limiting distribution of Cramér's maximal "prime" gaps is indeed the Gumbel distribution. Computational evidence suggests that the Gumbel distribution is also the a.s. limit law for record gaps in Cramér's model; we will discuss the distribution of records elsewhere.

\section{Cramér's Probabilistic Model of Primes}

Cramér's probabilistic model of primes is well known-and much criticized (Granville, 1995; Pintz, 2007).

\subsection{Setting up the Model}

Cramér (1936) sets up the model of primes as follows:

With respect to the ordinary prime numbers, it is well known that, roughly speaking, we may say that the chance that a given integer $n$ should be a prime is approximately $\frac{1}{\log n}$. This suggests that by considering the following series of independent trials we should obtain sequences of integers presenting a certain analogy with the sequence of ordinary prime numbers $p_{n}$.

Let $U_{1}, U_{2}, U_{3}, \ldots$ be an infinite series of urns containing black and white balls, the chance of drawing a white ball from $U_{n}$ being $\frac{1}{\log n}$ for $n>2$, while the composition of $U_{1}$ and $U_{2}$ may be arbitrarily chosen. We now assume that one ball is drawn from each urn, so that an infinite series of alternately black and white balls is obtained. If $P_{n}$ denotes the number of the urn from which the $n$-th white ball in the series was drawn, the numbers $P_{1}, P_{2}, \ldots$ will form an increasing sequence of integers, and we shall consider the class $C$ of all possible sequences $\left(P_{n}\right)$. Obviously the sequence $S$ of ordinary prime numbers $\left(p_{n}\right)$ belongs to this class.

We shall denote by $\Pi(x)$ the number of those $P_{n}$ which are $\leq x$, thus forming an analogy to the ordinary notation $\pi(x)$ for the number of primes $p_{n} \leq x$. (Cramér, 1936, pp. 25-26)

Cramér's model, as stated, is underdetermined: the content of urns $U_{1}$ and $U_{2}$ is arbitrary. To compute exact distributions of maximal gaps, we will assume that

(i) urn $U_{1}$ is empty-it produces neither "primes" nor "composites";

(ii) urn $U_{2}$ always produces white balls (i. e. the number 2 is certain to be "prime"). 


\subsection{Cramér's Results}

Using his probabilistic model of primes, Cramér obtained the following results for random primes $P_{k}$ :

- The expected value of $\Pi(x)$ (the number of RPs $\leq x$ ) is asymptotic to li $x: E \Pi(x) \sim$ li $x$ as $x \rightarrow \infty$.

- With probability $=1$, we have: $\limsup _{x \rightarrow \infty} \frac{|\Pi(x)-\operatorname{li} x|}{\sqrt{2 x \log \log x / \log x}}=1$.

- With probability $=1$, we have: $\limsup _{k \rightarrow \infty} \frac{P_{k+1}-P_{k}}{\log ^{2} P_{k}}=1$.

The latter result, restated for true primes $p_{k}$, constitutes the well-known Cramér's conjecture. The conjecture appears likely to be true. Indeed, computations of Oliveira e Silva, Herzog, and Pardi (2014) have verified that

$$
\begin{aligned}
& p_{k+1}-p_{k}<\log ^{2} p_{k} \text { for all primes } p_{k} \notin\{2,3,7\} \text { up to } 4 \times 10^{18}, \\
& p_{k}-p_{k-1}<\log ^{2} p_{k} \text { for all primes up to } 4 \times 10^{18} .
\end{aligned}
$$

At the same time, there exist large primes $p_{k}$ for which $0.8<\frac{p_{k+1}-p_{k}}{\log ^{2} p_{k}}<1$ (Sloane, 2014, A111943).

\section{Maximal Gaps Between Cramér's Random Primes}

\subsection{The Exact Distribution of Maximal Gaps}

We continue where Cramér left off. To obtain exact distributions of maximal gaps between Cramér's random primes, for now we will restrict ourselves to finite sets of $n$ consecutive urns $U_{1}, U_{2}, \ldots, U_{n}$. When our set of urns is small, we can compute the exact distributions of maximal gaps by hand, even without a computer.

For example, for $n=3$ we have only three urns: $U_{1}, U_{2}, U_{3}$. Of these, only $U_{3}$ produces random results:

- a white ball (RP) with probability $\frac{1}{\log 3} \approx 0.91$ or

- a black ball (RC) with probability $1-\frac{1}{\log 3} \approx 0.09$.

Thus, for the longest run of RCs, $R_{3}$, we have $R_{3}=0$ with probability 0.91 , and $R_{3}=1$ with probability 0.09 . Consequently, for the maximal gap between RPs, we have $G_{3}=1$ with probability 0.91 , and $G_{3}=2$ with probability 0.09 (Recall that, by definition, $G_{n}=R_{n}+1$ ).

One can visualize the exact distributions of maximal gaps in the form of histograms. With the help of a computer, we can find the exact distributions up to, say, $n=250$ urns. Figure 1 shows the respective computer-generated distributions of maximal gaps (cf. Schilling, 1990, pp. 197-204).

\subsection{The Limiting Distribution of Maximal Gaps}

In Figure 1, the exact distributions (histograms) of maximal gaps between RPs appear to approach the pdf curves of the Gumbel distribution with scale $\alpha_{n}=n / \operatorname{li} n$ and mode $\mu=\alpha_{n} \log \operatorname{li} n$. We can restate this observation in a more precise form:

Theorem 1 In Cramér's model with $n$ urns, the Gumbel distribution $\exp \left(-e^{-z}\right)$ is the limiting distribution of maximal gaps $G_{n}$ between RPs: there exist $a_{n}>0$ and $b_{n}$ such that

$$
\lim _{n \rightarrow \infty} P\left(G_{n} \leq x \equiv a_{n} z+b_{n}\right)=\exp \left(-e^{-z}\right), \quad \text { where } a_{n} \sim \alpha_{n}=\frac{n}{\operatorname{li} n}, \quad b_{n} \sim \alpha_{n} \log \operatorname{li} n .
$$

Equivalently, for $R_{n}$ (the longest runs of RCs) we have $\lim _{n \rightarrow \infty} P\left(R_{n} \leq a_{n} z+b_{n}\right)=\exp \left(-e^{-z}\right)$.

We will sketch two proofs of Theorem 1. The first proof will use the following lemmas.

Lemma of Common Median Suppose two Gumbel distributions have a common median and different scales $a \pm \varepsilon$, where $0<\varepsilon<\frac{a}{2}$. Then the cdfs of these Gumbel distributions differ by no more than $\frac{\varepsilon}{a}$.

Lemma of Common Scale Suppose two Gumbel distributions have a common scale $a>0$ and medians $M \pm \delta$. Then these Gumbel cdfs differ by no more than $\frac{\delta}{a}$.

Denote by $F_{n}(x)$ the cdf of the exact distribution of maximal gaps in Cramér's model with $n$ urns $U_{1}, \ldots, U_{n}$. Denote by $I_{n}(n \geq 10)$ the largest interval of the $x$ axis such that $F_{n}(x) \in\left[(\log n)^{-1}, 1-(\log n)^{-1}\right]$ for all $x \in I_{n}$ (One can show that $\left.I_{n} \subset\left[\log n, \log ^{2} n\right]\right)$. 
Squeeze Lemma Let $M_{n}$ be the median of $F_{n}(x)$. Then $F_{n}(x)$ is squeezed in the area bounded by the Gumbel distribution $c d f$ with medians $M=M_{n} \pm \delta$ and scales $a=n / \operatorname{li} n \pm \varepsilon$ :

$$
\min _{\substack{M=M_{n} \pm \delta \\ a=n / \operatorname{li} n \pm \varepsilon}} 2^{-e^{-\frac{x-M}{a}}} \leq F_{n}(x) \leq \max _{\substack{M=M_{n} \pm \delta \\ a=n / \operatorname{lin} n \pm \varepsilon}} 2^{-e^{-\frac{x-M}{a}}} \quad \text { for } n \geq 10, x \in I_{n},
$$

where one can take $\varepsilon=3 / 2, \delta=1$ (See Figure 2. The sequence $\left\{M_{n}\right\}$ is OEIS A235492, Sloane, 2014).

Hereafter we often use these formulas expressing the Gumbel distribution cdf in terms of its scale $a$, mode $\mu$ and median $M$ :

$\operatorname{Gumbel}(x ; a, \mu)=e^{-e^{-\frac{x-\mu}{a}}}=2^{-e^{-\frac{x-M}{a}}}, \quad$ where $M=\mu-a \log \log 2 \approx \mu+0.3665 a$.
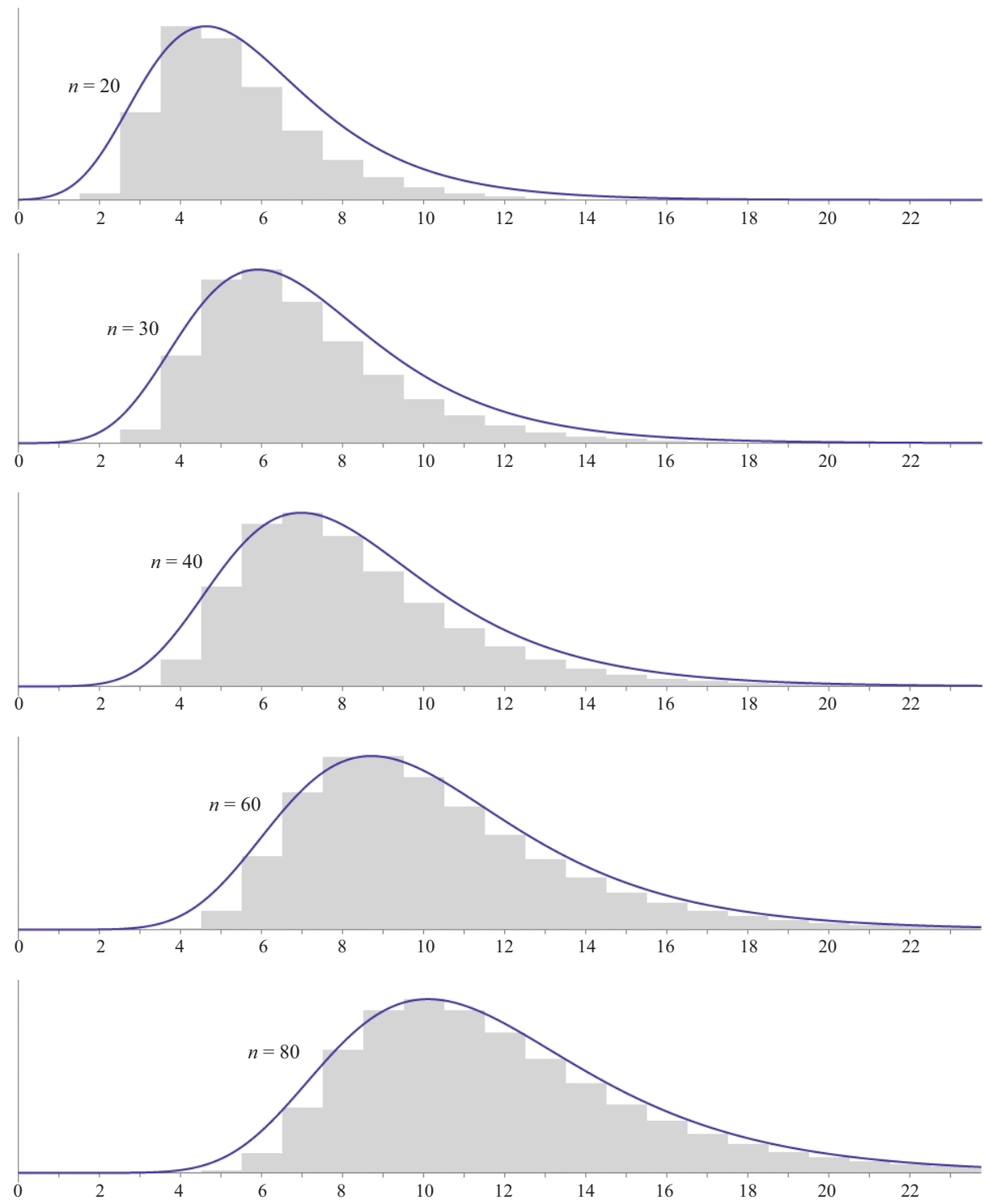

Figure 1. Histograms of the exact distributions of maximal prime gaps in Cramer's model with $n$ urns, computed for $n=20,30,40,60,80$. The smooth curves represent Gumbel distributions (pdf) with the scale $a=n /$ li $n$ and mode $\mu=n \log (\operatorname{li} n) / \operatorname{li} n$. Interested readers can compute and plot the exact distributions of maximal gaps online at http://www . javascripter . net/math/statistics/maximalprimegapsincramermodel.htm 


\subsubsection{First Proof of Theorem 1}

Suppose the number of urns $n$ is large. We will examine two cases: (a) $x \in I_{n}$; (b) $x \notin I_{n}$.

Case (a) First, we consider $F_{n}(x)$ for $x \in I_{n}$, i. e., $(\log n)^{-1} \leq F_{n}(x) \leq 1-(\log n)^{-1}$.

Let us estimate $M_{n}$, the median of $F_{n}(x)$. As before, let $\alpha_{n}=n /$ li $n$ and $\varepsilon=3 / 2$. We can approximate Cramér's proportions of white balls in urns using two different procedures:

(i) set the white-to-black-balls ratio in all urns to $\frac{1}{\alpha_{n}+\varepsilon}$, then increase the percentages of white balls in all urns or, alternatively,

(ii) set the white-to-black-balls ratio in all urns to $\frac{1}{\alpha_{n}-\varepsilon}$, then reduce the percentages of white balls in all but a small subset of urns.

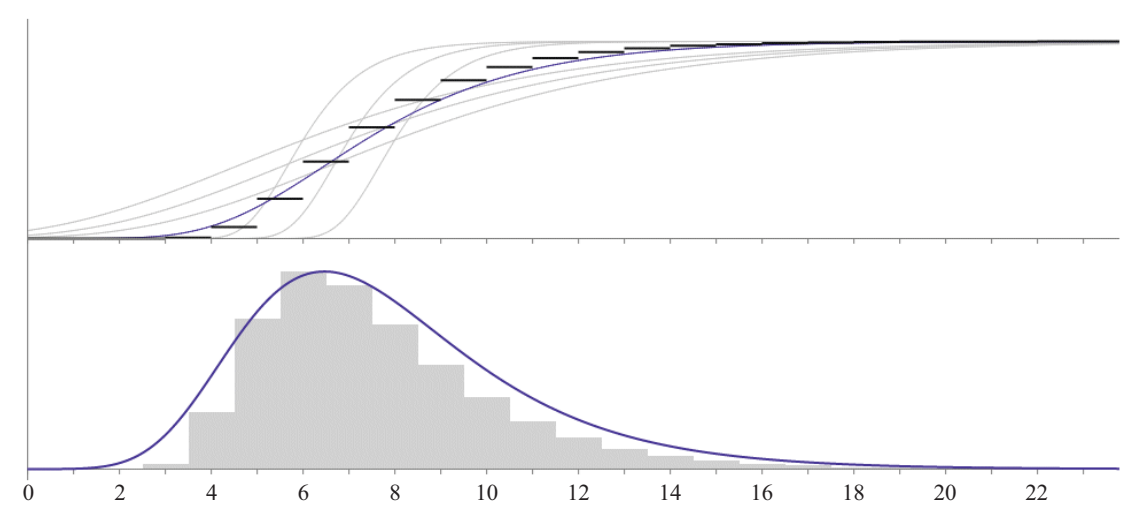

Figure 2. Bottom: the histogram of maximal prime gaps in Cramér's model with 35 urns. Top: the black "staircase" is the exact cdf of the distribution of maximal prime gaps in Cramér's model with 35 urns. The dark curve shows the corresponding Gumbel distribution with scale $a=n / \operatorname{li~} n$ and mode $\mu=n \log (\operatorname{li} n) / \operatorname{li} n$. Light gray curves illustrate the Gumbel cdfs with scales $a=n / \operatorname{li} n \pm \varepsilon$ and medians $M=M_{n} \pm 1$ used in Squeeze Lemma; $n=35$

Note that the white-to-black balls ratio $1 / a$ can be approximated by the exponential distribution of gaps $\operatorname{Exp}(x ; a)$. Observe also that increasing the percentage of white balls pushes the median of maximal gaps to the left, while reducing this percentage pushes the median to the right. Therefore, $M_{n}$ must be somewhere between the medians of $\left(\operatorname{Exp}\left(x ; \alpha_{n}-\varepsilon\right)\right)^{\mathrm{li} n}$ and $\left(\operatorname{Exp}\left(x ; \alpha_{n}+\varepsilon\right)\right)^{\mathrm{li} n}$ :

$$
\text { median }\left(\operatorname{Exp}\left(x ; \alpha_{n}-\varepsilon\right)\right)^{\operatorname{li} n} \lesssim M_{n} \lesssim \operatorname{median}\left(\operatorname{Exp}\left(x ; \alpha_{n}+\varepsilon\right)\right)^{\operatorname{li} n} .
$$

Since $\alpha_{n} \sim \log n$ while $\varepsilon=O(1)$, the above lower and upper bounds are asymptotic to each other and to the median of $\left(\operatorname{Exp}\left(x ; \alpha_{n}\right)\right)^{\text {lin }}$, so we must have

$$
M_{n} \sim \operatorname{median}\left(\operatorname{Exp}\left(x ; \alpha_{n}\right)\right)^{\operatorname{li} n} \quad \text { as } n \rightarrow \infty .
$$

That is to say, as $n \rightarrow \infty$, the median $M_{n}$ of $F_{n}(x)$ must be asymptotic to the median of the limiting distribution of maxima of $\lfloor\operatorname{li} n\rfloor$ i.i.d. random variables with the exponential distribution $\operatorname{Exp}\left(x ; \alpha_{n}\right)$. But this limiting distribution is precisely the Gumbel distribution Gumbel $\left(x ; \alpha_{n}, \alpha_{n} \log \operatorname{li} n\right)$ (Gumbel, 1935; Gnedenko, 1943; Hall \& Wellner, 1979); therefore

$$
M_{n} \sim \operatorname{median}\left(\operatorname{Gumbel}\left(x ; \alpha_{n}, \alpha_{n} \log \operatorname{li} n\right)\right) \approx \alpha_{n} \log \operatorname{li} n+0.3665 \alpha_{n} .
$$

On the other hand, it follows from Lemmas that $F_{n}(x)$ is squeezed in the $\Delta_{n}$-neighborhood of the Gumbel cdf $2^{-e^{-\frac{k-M_{n}}{\alpha_{n}}}}$, where $\varepsilon$ and $\delta$ are defined as in Squeeze Lemma,

$$
\Delta_{n}=\frac{\varepsilon+\delta}{\alpha_{n}}, \quad \text { and } \quad \alpha_{n}=\frac{n}{\operatorname{li} n} \sim \log n \rightarrow \infty \quad \text { as } n \rightarrow \infty .
$$

It is easy to see that $\Delta_{n} \rightarrow 0$ as $n \rightarrow \infty$. 
To sum it up: the median $M_{n}$ is asymptotic to the median of $\operatorname{Gumbel}\left(x ; \alpha_{n}, \alpha_{n} \log \operatorname{li} n\right)$, while the limiting shape of $F_{n}(x)$ is dictated by the fact that $F_{n}(x)$ is in the $\Delta_{n}$-neighborhood of the Gumbel cdf $2^{-e^{-\frac{x-M_{n}}{\alpha_{n}}}}$, with $\lim _{n \rightarrow \infty} \Delta_{n}=0$. This completes the proof for case (a).

Case (b) Now consider $x \notin I_{n}$ : either $F_{n}(x)<(\log n)^{-1}$ or $F_{n}(x)>1-(\log n)^{-1}$. Taking into account the monotonicity of cdfs, we can conclude from Lemmas that, for large $n$,

$$
\begin{aligned}
2^{-e^{-\frac{x-M_{n}}{\alpha_{n}}}} & <\frac{\varepsilon+\delta}{\alpha_{n}}+(\log n)^{-1} \quad \text { if } \quad F_{n}(x)<(\log n)^{-1}, \\
1-2^{-e^{-\frac{x-M_{n}}{\alpha_{n}}}} & <\frac{\varepsilon+\delta}{\alpha_{n}}+(\log n)^{-1} \quad \text { if } 1-F_{n}(x)<(\log n)^{-1},
\end{aligned}
$$

where $\alpha_{n}=\frac{n}{\operatorname{li} n} \sim \log n$. Therefore, $\left|F_{n}(x)-2^{-e^{-\frac{x-M_{n}}{\alpha_{n}}}}\right|=O\left((\log n)^{-1}\right) \rightarrow 0$ as $n \rightarrow \infty$.

\subsubsection{Second Proof of Theorem 1}

We begin by proving the theorem for longest runs $R_{n}$ of “composites”. In Cramér's model, urns $U_{n}(n \geq 3)$ produce white balls with a monotonically decreasing, slowly varying probability $1 / \log n \rightarrow 0$ as $n \rightarrow \infty$. We observe that the more general Theorem A.1 (see Appendix) is applicable to our situation. Theorem A.1 tells us that, as $n \rightarrow \infty$, the limiting distribution of longest runs exists; it is the Gumbel distribution with the scale and mode parameters determined by $E \Pi(n)$, the expected total number of RPs $\leq n$ :

$$
\begin{aligned}
\text { scale } a_{n} & \sim \frac{n}{E \Pi(n)}=\frac{n}{\operatorname{li} n+O(1)} \sim \frac{n}{\operatorname{li} n} \\
\text { mode } b_{n} & \sim \frac{n \log E \Pi(n)}{E \Pi(n)}=\frac{n}{\operatorname{li} n+O(1)} \log (\operatorname{li} n+O(1)) \sim \frac{n}{\operatorname{li} n} \log \operatorname{li} n .
\end{aligned}
$$

Here we have used the fact that, for $n \geq 3$, the expected total number of RPs $\leq n$ is

$$
E \Pi(n)=1+\sum_{k=3}^{n} \frac{1}{\log k}=\operatorname{li} n+O(1) \quad \text { as } n \rightarrow \infty .
$$

Thus we can use the above scale and mode as rescaling parameters $a_{n}$ and $b_{n}$, to obtain the standard Gumbel distribution $\exp \left(-e^{-z}\right)$. Note that $1 \ll a_{n} \ll b_{n}$ as $n \rightarrow \infty$; so the distribution rescaling formula $z=\frac{x-b_{n}}{a_{n}}$ will produce approximately equal values of $z$, no matter whether we are rescaling the longest runs $R_{n}$ or maximal gaps $G_{n} \equiv R_{n}+1$.

\subsection{Properties of the Distribution of Maximal Gaps}

Let us look at the the properties of the distribution of maximal gaps. We can readily see that the exact distribution of maximal gaps is discrete and bounded, while the limiting Gumbel distribution is continuous, smooth, and unbounded. Below we discuss two properties that are common to the exact and asymptotic distributions of maximal gaps: log-concavity and unimodailty.

\subsubsection{Log-Concavity}

A function $f(x)>0$ with a convex domain is log-concave if $\log f(\lambda x+(1-\lambda) y) \geq \lambda \log f(x)+(1-\lambda) \log f(y)$ for all $0 \leq \lambda \leq 1$ and for all $x, y$ in the domain of $f(x)$. A discrete sequence $\left\{s_{k}\right\}$ is log-concave if $s_{k}^{2} \geq s_{k-1} s_{k+1}$ for each middle term $s_{k}$.

Log-concavity of the limiting distribution of maximal gaps. It is well known (and easy to check given the formulas for the distribution's pdf and cdf) that the Gumbel distribution pdf and cdf are log-concave. Note that, in general, if $f(x)$ is a continuous distribution pdf and $F(x)$ is the corresponding cdf, then the following implications are true:

$$
\begin{aligned}
& f(x) \text { is log-concave } \Rightarrow F(x) \text { is log-concave, } \\
& f(x) \text { is log-concave } \Leftrightarrow 1-F(x) \text { is log-concave. }
\end{aligned}
$$

Log-concavity of exact distributions of maximal gaps. A direct computational check shows that all the exact distribution functions we have computed in Section 4.1 are also log-concave:

$$
F_{n}(k)^{2} \geq F_{n}(k-1) F_{n}(k+1), \quad 1<k<n .
$$


However, the log-concavity is not necessarily preserved if we use an approximate (e.g. Monte-Carlo) algorithm rather than the exact formulas for computing the finite distribution functions $F_{n}(x)$.

\subsubsection{Unimodality}

The Gumbel distribution $\operatorname{Gumbel}(x ; a, \mu)$ is unimodal for any $\mu$ and any $a>0$ : it has a unique mode (most probable value), namely, $\mu$. What about the exact distribution of maximal gaps between RPs in Cramér's model with $n$ urns? By Theorem 1, the Gumbel distribution is the limiting distribution of maximal gaps between RPs in Cramér's model; therefore it is reasonable to expect that, for large $n$, in Cramér's model with $n$ urns the exact distribution of maximal gaps between RPs is also unimodal. On the other hand, for moderate values of $n$ one can check by direct computation that each of the exact distributions of maximal gaps between RPs is unimodal. Here we will state without a formal proof the following

Unimodality Lemma In Cramér's model with $n$ urns, the exact distribution of maximal gaps between RPs is unimodal for each $n>1$.

\section{Generalizations • Applications • Concluding Remarks}

We have thus shown that the Gumbel distribution is the limit law for extreme gaps between Cramér's random primes. In particular, when the number of urns $n$ in Cramér's model is large, the distribution of maximal prime gaps approaches the Gumbel distribution $\operatorname{Gumbel}\left(x ; a_{n}, b_{n}\right)$ with these parameters:

$$
\text { scale } a_{n} \sim \frac{n}{\operatorname{li} n}, \quad \text { mode } b_{n} \sim \frac{n}{\operatorname{li} n} \log \operatorname{li} n
$$

In view of this limit law for maximal gaps between Cramér's RPs $\leq n$, additional questions naturally arise:

- Is the Gumbel distribution, after proper rescaling, also an (almost sure) limit law for record prime gaps observed in a single infinite sequence of Cramér's random primes? Cf. Resnick (1973) and Arnold et al. (1998, p. 193).

- Is the Gumbel distribution, after proper rescaling, also the limit law for record gaps between true primes?

While the latter question appears particularly difficult, it clearly suggests that our probabilistic result has a potential application to number theory. Indeed, in the spirit of Cramér's program we quoted in Introduction, a numbertheoretic result can be first obtained heuristically via probability considerations, and a rigorous number-theoretic proof could be found afterwards.

A straightforward generalization extends the Gumbel limit law to maximal gaps between prime constellationsdense clusters of consecutive primes with a repeatable pattern (see Table 1). For this generalization, it is essential that we regard prime constellations near $x$ as random events occurring with a slowly varying probability decreasing to zero as $x \rightarrow \infty$. Just as with extreme gaps between primes, here we do not have rigorous number-theoretic proofs-but we do have a convincing probabilistic argument explained below.

Table 1. Prime constellations. Conjectured probabilities to find a constellation starting at $x$ (cf. Forbes, 2012)

\begin{tabular}{lcc}
\hline Constellation type & Pattern of primes & Conjectured probability $C(\log x)^{-K}$ at $p \approx x$ \\
\hline Twin primes & $\{p, p+2\}$ & $1.32032(\log x)^{-2}$ \\
Prime triplets & $\{p, p+2, p+6\}$ & $2.85825(\log x)^{-3}$ \\
Prime triplets & $\{p, p+4, p+6\}$ & $2.85825(\log x)^{-3}$ \\
Prime quadruplets & $\{p, p+2, p+6, p+8\}$ & $4.15118(\log x)^{-4}$ \\
Prime quintuplets & $\{p, p+2, p+6, p+8, p+12\}$ & $10.13179(\log x)^{-5}$ \\
Prime quintuplets & $\{p, p+4, p+6, p+10, p+12\}$ & $10.13179(\log x)^{-5}$ \\
Prime sextuplets & $\{p, p+4, p+6, p+10, p+12, p+16\}$ & $17.29861(\log x)^{-6}$ \\
\hline
\end{tabular}

The idea to model prime constellations by random events with predictable probabilities stems from the $K$-tuple conjecture of Hardy and Littlewood (1922). Riesel (1994, pp. 60-68) gives an accessible account of this conjecture. Forbes (2012) published extensive numerical data on densest permissible prime constellations; Table 1 is based on a small subset of Forbes' data. Once we agree to treat prime constellations as random events with slowly varying probabilities given in Table 1, the Gumbel limit law for maximal gaps between such events immediately follows from Theorem A.1 (Appendix). Specifically, we can estimate the scale and mode in the limit law Gumbel $\left(x ; a_{n}, b_{n}\right)$ for a particular constellation by expressing them in terms of $E \Pi_{c}(n)$, the expected total count of constellations 
below $n$ :

$$
\text { scale } a_{n} \sim \frac{n}{E \Pi_{c}(n)}, \quad \operatorname{mode} b_{n} \sim \frac{n \log E \Pi_{c}(n)}{E \Pi_{c}(n)}, \quad E \Pi_{c}(n) \approx C \int_{0}^{n}(\log x)^{-K} d x,
$$

where the values of $C$ and $K$ are given in the last column of Table 1. Thus prime constellations can be simulated using a modified version of Cramér's model: urn $U_{n}$ produces a white ball with probability $C(\log n)^{-K}$. (Of course, it is also possible to simulate prime constellations by $K$ consecutive white balls in the classical Cramér model as described in Section 3. However, the latter approach is inferior because it does not accommodate the known values of the Hardy-Littlewood constants $C$ in Table 1).

The generalization of the Gumbel limit law to extreme gaps between prime sextuplets (Table 1, last line) leads to a somewhat unexpected application: the riecoin cryptocurrency (Riecoin.org, 2014). The computational process of riecoin mining consists in finding very large prime sextuplets (over 300 bits in size). The Gumbel limit law for maximal gaps between prime sextuplets therefore also describes the distribution of "worst cases" in terms of computational work in riecoin mining.

To conclude our brief discussion of applications, let us quote the British mathematician G. H. Hardy (1877-1947) who made spectacular breakthroughs in the study of prime numbers-and paradoxically cautioned against emphasizing the applications and practical usefulness of mathematics:

A science is said to be useful if its development tends to accentuate the existing inequalities in the distribution of wealth, or more directly promotes the destruction of human life. The theory of prime numbers satisfies no such criteria. Those who pursue it will, if they are wise, make no attempt to justify their interest in a subject so trivial and so remote, and will console themselves with the thought that the greatest mathematicians of all ages have found in it a mysterious attraction impossible to resist (Hardy, 1915).

\section{Acknowledgements}

The author expresses his sincere gratitude to the anonymous referees for their time and attention to this manuscript and for constructive criticism. Special thanks also to Marek Wolf, Luis Rodriguez and Carlos Rivera whose ideas and conjectures served as starting points for this work (cf. Wolf, 2011; Rodriguez \& Rivera, 2009).

\section{References}

Anderson, C. W. (1970). Extreme value theory for a class of discrete distributions with applications to some stochastic processes. J. Appl. Prob., 7, 99-113. http://dx.doi.org/10.2307/3212152

Arnold, B. C., Balakrishnan, N., \& Nagaraja, H. N. (1998). Records. New York, NY: Wiley. http://dx.doi.org/10.1002/9781118150412

Caldwell, C. (2010). The gaps between primes. Section 3: Table and graph of record gaps. Retrieved from http://primes.utm.edu/notes/gaps.html\#table

Cramér, H. (1936). On the order of magnitude of the difference between consecutive prime numbers. Acta Arith., 2, 23-46. Retrieved from http://matwbn.icm.edu.pl/ksiazki/aa/aa2/aa212.pdf

De Haan, L., \& Ferreira, A. (2006). Extreme Value Theory. New York, NY: Springer.

Fisher, R. A., \& Tippett, L. H. C. (1928). Limiting forms of the frequency distribution of the largest and smallest member of a sample. Math. Proc. Cambridge Philos. Soc., 24, 180-190. http://dx.doi.org/10.1017/S0305004100015681

Forbes, A. D. (2012). Prime k-tuplets. Section 21: List of all possible patterns of prime $k$-tuplets. The HardyLittlewood constants pertaining to the distribution of prime $k$-tuplets. Retrieved from http://anthony.d.forbes.googlepages.com/ktuplets.htm

Gnedenko, B. V. (1943). Sur la distribution limite du terme maximum d'une série aléatoire. Ann. Math., 44, 423453. English translation: On the limiting distribution of the maximum term in a random series. In S. Kotz, \& N. L. Johnson (Eds.) (1993). Breakthroughs in Statistics, 1. Foundations and Basic Theory (pp. 185-225). New York, NY: Springer. http://dx.doi.org/10.2307/1968974

Granville, A. (1995). Harald Cramér and the distribution of prime numbers. Scand. Actuar. J., 1, 12-28. http://dx.doi.org/10.1080/03461238.1995.10413946 
Gumbel, E. J. (1935). Les valeurs extrêmes des distributions statistiques. Annales de l'institut Henri Poincaré, 5 , 115-158. Retrieved from http://archive.numdam.org/article/AIHP\%5F1935\%5F\%5F5\%5F2\%5F115\%5F0.pdf

Gumbel, E. J. (1958). Statistics of Extremes. New York, NY: Columbia University Press. Mineola, NY: Dover.

Hall, W. J., \& Wellner, J. A. (1979). The rate of convergence in law for the maximum of an exponential sample. Statist. Neerlandica, 33, 151-154. http://dx.doi.org/10.1111/j.1467-9574.1979.tb00671.x

Hardy, G. H. (1915). Prime numbers. In Reports on the State of Science. The 85th Meeting of the British Association for the Advancement of Science, Manchester, 1915 (pp. 350-355). Reprinted in P. Borwein, S. Choi, B. Rooney, \& A. Weirathmueller (Eds.) (2008). The Riemann Hypothesis. A Resource for the Afficionado and Virtuoso Alike, 27 (pp. 301-306). CMS Books in Mathematics. New York, NY: Springer.

Hardy, G. H., \& Littlewood, J. E. (1922). Some problems of 'Partitio Numerorum.' III. On the expression of a number as a sum of primes. Acta Math., 44, 1-70. http://dx.doi.org/10.1007/BF02403921

Kourbatov, A. (2013). Maximal gaps between prime $k$-tuples: a statistical approach. J. Integer Sequences, 16, Article 13.5.2. Retrieved from https://cs.uwaterloo.ca/journals/JIS/voll6.html http://arxiv.org/abs/1301.2242

Mladenović, P. (1999). Limit theorems for the maximum terms of a sequence of random variables with marginal geometric distributions. Extremes, 2(4), 405-419. http://dx.doi.org/10.1023/A:1009952232519

Nevzorov, V. B. (2001). Records: Mathematical Theory, AMS Translations Series 194, Providence, RI: AMS.

Nicely, T. R. (2013). First occurrence prime gaps. Retrieved from http://www.trnicely.net/gaps/gaplist.html

Oliveira e Silva, T., Herzog, S., \& Pardi, S. (2014). Empirical verification of the even Goldbach conjecture and computation of prime gaps up to $4 \cdot 10^{18}$. Math. Comp., 83 (to appear).

Pintz, J. (2007). Cramér vs. Cramér: On Cramér's probabilistic model of primes. Funct. Approx. Comment. Math., 37, 361-376. http://dx.doi.org/10.7169/facm/1229619660

Resnick, S. I. (1973). Record values and maxima. The Annals of Probability, 1, 650-662. http://dx.doi.org/10.1214/aop/1176996892

Riecoin.org (2014). Riecoin: a cryptocurrency using prime sextuplets. Retrieved from http://www.riecoin.org

Riesel, H. (1994). Prime Numbers and Computer Methods for Factorization (2nd ed.). Boston, MA: Birkhäuser.

Rodriguez, L., \& Rivera, C. (2009). Conjecture 66. Gaps between consecutive twin prime pairs. Retrieved from http://www.primepuzzles.net/conjectures/

Schilling, M. F. (1990). The longest run of heads. College Math. J., 21, 196-207. http://dx.doi.org/10.2307/2686886

Shanks, D. (1964). On maximal gaps between successive primes. Math. Comp., 18, 646-651. http://dx.doi.org/10.1090/S0025-5718-1964-0167472-8

Sloane, N. J. A. (Ed.) (2014). The On-Line Encyclopedia of Integer Sequences. Published electronically at: http://oeis.org/A235492

Wolf, M. (2011). Some heuristics on the gaps between consecutive primes. preprint. Retrieved from: http://arxiv.org/abs/1102.0481

\section{Appendix: Maximal Intervals Between Random Events Occurring With Slowly Varying Probability $\ell(t) \rightarrow 0$}

In this appendix we give a general theorem whose particular case $(\ell(t)=1 / \log t$ for $t \geq 3)$ has been used in Section 4.2.2. We say that a function $\ell(t)>0$ is slowly varying if it is defined for positive $t$, and $\lim _{t \rightarrow \infty} \frac{\ell(\lambda t)}{\ell(t)}=1$ for any fixed $\lambda>0$ (de Haan \& Ferreira, 2006, p. 362).

Theorem A.1 Consider biased coins with tails probability $\ell(k)$ at the $k$-th toss, $0<\ell(k)<1$, where $\ell(t)$ is a smooth, slowly varying, monotonically decreasing function, and $\lim _{t \rightarrow \infty} \ell(t)=0$. Then, after a large number $n$ of tosses, the asymptotic distribution of the longest runs of heads $R_{n}$ is the Gumbel distribution: there exist $a_{n}>0$ and $b_{n}$ such that

$$
\lim _{n \rightarrow \infty} P\left(R_{n} \leq x \equiv a_{n} z+b_{n}\right)=\exp \left(-e^{-z}\right), \quad \text { where } a_{n} \sim \frac{n}{E \Pi(n)}, \quad b_{n} \sim \frac{n \log E \Pi(n)}{E \Pi(n)} .
$$


Here $E \Pi(n)$ is the mathematical expectation of the total number of tails $\Pi(n)$ observed during the first $n$ tosses:

$$
E \Pi(n)=\sum_{k=1}^{n} \ell(k)
$$

It may be surprising that the asymptotic distribution does exist at all for discrete events occurring with a slowly varying probability $\ell(t) \rightarrow 0$. In contrast, for a biased coin with a constant positive probability of tails, the limiting distribution of the longest run of heads does not exist (Schilling, 1990, p. 203). In the lemmas below, we assume the conditions of Theorem A.1 and take $\lambda$ to be arbitrarily large $(\lambda \gg 1)$. The lemmas are easy to prove using the theory of regularly varying functions (see, e.g., de Haan \& Ferreira, 2006, pp. 361-367).

Lemma $1 \lim _{t \rightarrow \infty} \Pi(t)=\infty$ a.s., while $\lim _{t \rightarrow \infty} \frac{E \Pi(t)}{t}=0$.

Lemma $2 \lim _{t \rightarrow \infty} E(\Pi(\lambda t)-\Pi(t))=\infty$.

Lemma $3 \lim _{t \rightarrow \infty}(E \Pi(\lambda t) / E \Pi(t))=\lambda$.

Lemma $4 \lim _{t \rightarrow \infty} \frac{E(\Pi(\lambda t)-\Pi(t))}{E \Pi(\lambda t)}=\frac{\lambda-1}{\lambda}$.

Proof of Theorem A.1. Let $k$ denote the ordinal number of a coin toss, so the $k$-th toss has the tails probability $\ell(k)$. Take $\lambda \gg 1$ and consider the sequence $S(t, \lambda t)$ of consecutive tosses with $t<k \leq \lambda t$. For toss sequences $S(t, \lambda t)$ with larger and larger $t$, we see that:

(i) $\ell(k)$ becomes nearly constant: $\ell(\lambda t) \lesssim \ell(k) \lesssim \ell(t)$ (by monotonicity + slow variation);

(ii) the expected total number of tails is $E(\Pi(\lambda t)-\Pi(t)) \rightarrow \infty$ as $t \rightarrow \infty$ (by Lemma 2).

As $t$ grows larger, the outcome of the toss sequence $S(t, \lambda t)$ becomes indistinguishable from an equally long sequence of tosses of a constant-bias coin whose tails probability $q$ is

$$
q=\frac{E(\Pi(\lambda t)-\Pi(t))}{\lambda t-t} \approx \frac{E \Pi(\lambda t)}{\lambda t} \approx \frac{E \Pi(n)}{n}, \quad \text { where we set } n=\lfloor\lambda t\rfloor .
$$

In the latter setup with a constant-bias coin, head runs are modeled by i.i.d. geometric random variables (Schilling, 1990). The expected total number $m$ of head runs is

$$
m=q(\lambda t-t)=E(\Pi(\lambda t)-\Pi(t)) \approx \frac{\lambda-1}{\lambda} E \Pi(n), \quad \text { with } n=\lfloor\lambda t\rfloor,
$$

In turn, these $m$ geometric i.i.d. variables are highly accurately approximated by exponential i.i.d. variables whose common cdf is $\operatorname{Exp}(\xi ; a)=1-e^{-\xi / a}$ (Anderson, 1970). The scale parameter $a$ in $\operatorname{Exp}(\xi ; a)$ is given by

$$
a=\frac{\lambda t-t}{E(\Pi(\lambda t)-\Pi(t))} \approx \frac{n}{E \Pi(n)} \quad \text { (here and above we have used Lemma 4). }
$$

The largest value $L$ of $m$ exponential i.i.d.random variables, with the cdf $1-e^{-\xi / a}$, has the limiting Gumbel distribution (Gumbel, 1935; Gnedenko, 1943; Hall \& Wellner, 1979):

$$
\lim _{m \rightarrow \infty} P(L \leq a z+a \log m)=\exp \left(-e^{-z}\right)
$$

However, it is well known that for the geometrically distributed runs of heads with a constant-bias coin (i. e. the tails probability $q=$ const) the longest runs $R$ do not have a limiting distribution (Anderson, 1970; Schilling, 1990):

$$
P(R \leq a z+a \log m)=\exp \left(-e^{-z}\right)+O(q),
$$

with the geometric-to-exponential approximation error $O(q)$ preventing the convergence of the exact longest run distributions to the Gumbel distribution. Nevertheless, in our original setup with slowly varying bias of the coin, instead of a constant $q$ we have

$$
q_{n} \sim a_{n}^{-1} \sim \ell(n) \sim \frac{E \Pi(n)}{n} \rightarrow 0 \quad \text { as } n \rightarrow \infty .
$$


For the sequence $S(t, \lambda t)$ to contain the absolute longest run of heads $R_{n}$ up to $n=\lfloor\lambda t\rfloor$, we must take $\lambda$ very large, so $\frac{\lambda-1}{\lambda} \rightarrow 1$, and from Equation (1) we have $\log m \approx \log E \Pi(n)$. Now Equations (2) and (3) yield the limiting distribution of $R_{n}$ under the theorem conditions:

$$
\lim _{n \rightarrow \infty} P\left(R_{n} \leq a_{n} z+b_{n}\right)=\exp \left(-e^{-z}\right), \quad \text { where } a_{n} \sim \frac{n}{E \Pi(n)}, \quad b_{n} \sim \frac{n \log E \Pi(n)}{E \Pi(n)} .
$$

Thus the convergence to the limiting Gumbel distribution is restored. The convergence rate clearly depends on the actual choice of the slowly varying function $\ell(t) \rightarrow 0$.

Remarks. Theorem A.1 is general enough to describe maximal gaps for "primes" and "prime constellations" in Cramér's model. However, comparing it to the results of Mladenović (1999), one might look for a further generalization. For example, what kind of a limiting distribution (if any) would we get if in Theorem A.1 we replace the slowly varying probability $\ell(t) \rightarrow 0$ by some regularly varying probability $q(t) \rightarrow 0, q(t) \in \mathrm{RV}_{\alpha}$ with, say, $-1 \leq \alpha \leq 0$ ? Here $\alpha$ is the index of regular variation; for definitions of $\alpha$ and $\mathrm{RV}_{\alpha}$ see, e. g., de Haan \& Ferreira (2006, p. 362). Note that $\alpha=0$ corresponds to slowly varying functions.

\section{Copyrights}

Copyright for this article is retained by the author(s), with first publication rights granted to the journal.

This is an open-access article distributed under the terms and conditions of the Creative Commons Attribution license (http://creativecommons.org/licenses/by/3.0/). 